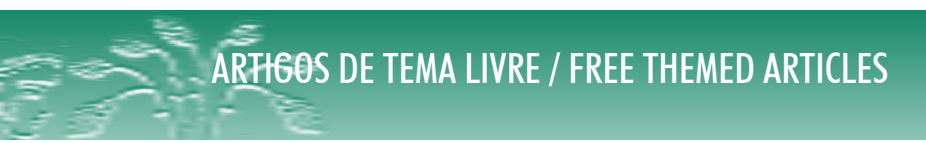

DOI: 10.12957/demetra.2017.28060

\title{
Consumo alimentar de crianças de seis a doze meses e perfil sociodemográfico materno
}

\section{Food intake by children aged six to twelve months and maternal sociodemographic profile}

\author{
Fernanda de Bona Coradi \\ Silvania Moraes Bottaro' \\ Vanessa Ramos Kirsten ${ }^{1}$ \\ 1 Universidade Federal de Santa Maria, \\ Departamento de Alimentos e Nutrição. Palmeira \\ das Missões-RS, Brasil. \\ Correspondência / Correspondence \\ Fernanda de Bona Coradi \\ E-mail: fernandacoradi@yahoo.com.br
}

\section{Resumo}

Objetivo: avaliar o consumo alimentar de crianças de 6 a 12 meses de idade e sua relação com variáveis sociodemográficas maternas, do município de Arvorezinha, RS. Método: estudo transversal com crianças que avaliou consumo alimentar no dia anterior por meio dos formulários do Sistema de Vigilância Alimentar e Nutricional (SISVAN) de 2008 e 2015 e variáveis maternas aferidas através de formulário desenvolvido pelos pesquisados, sendo as mães as entrevistadas. Resultados: verificouse alta frequência na ingesta de comida de sal $(95,31 \%)$, cereais $(90,48 \%)$, fruta $(87,30 \%)$ e legumes $(70,31 \%)$, e baixa frequência na ingesta de mingau com leite $(31,81 \%)$, verdura de folha $(15,64 \%)$, embutidos $(7,94 \%)$ e fígado $(4,76 \%)$. As variáveis maternas idade, direito à licença-maternidade, a escolaridade, a renda $\mathrm{e}$ ser beneficiário do programa Bolsa Família tiveram associação com consumo alimentar dos filhos. Discussão: os resultados encontrados são semelhantes a demais estudos publicados e têm forte relação com recomendações de uma Alimentação Saudável para Menores de Dois Anos. Conclusão: as crianças apresentaram consumo alimentar saudável, principalmente aos 9 meses, e o perfil sociodemográfico materno demonstrou relação com a alimentação dos filhos.

Palavras-chave: Consumo de Alimentos. Nutrição da Criança. Política Nutricional. 


\section{Abstract}

Objective: To evaluate food intake of children aged six to twelve months and its relationship with maternal sociodemographic variables in the town of Arvorezinha, state of Rio Grande do Sul, Brazil. Method: It is a cross-sectional study that analyzed children's food intake on the day before data collection, through data from the Food and Nutrition Surveillance System (SISVAN) in 2008 and 2015, and maternal variables evaluated through a form completed by the respondents and interviews with the participating mothers. Results: There was high frequency of intake of savory food $(95.31 \%)$, cereals $(90.48 \%)$, fruits $(87.30 \%)$ and vegetables $(70.31 \%)$, and low frequency of intake of porridge with milk (32.81\%), leafy vegetables (15.64\%), sausages $(7.94 \%)$ and liver (4.76\%). The maternal variables age, right to maternity leave, receiving an alowance from the Bolsa Familia cash transfer program were associated with children's food intake. Discussion: The results are similar to those of other published studies and are strongly related to the recommendations for healthy food intake by two-year-olds. Conclusion: the children in the present study have healthy food intake, especially at nine months, and maternal sociodemographic profile was related to children's food intake.

Keywords: Food Consumption. Child Nutrition. Nutrition Policy.

\section{Introdução}

Com o propósito de melhorar as condições de alimentação, nutrição e saúde da população brasileira, o Ministério da Saúde, na década de 1970, criou o Sistema de Vigilância Alimentar e Nutricional (SISVAN), a fim de avaliar e monitorar a situação alimentar e nutricional, detectando fatores de risco agravantes à saúde. ${ }^{1}$ Entretanto, somente na década de $1990^{1,2}$ foi implementado em âmbito nacional, com atuação até o momento. O sistema dispõe de protocolos para verificar e caracterizar a alimentação e o estado nutricional em determinadas faixas etárias de forma eficaz e representativa. ${ }^{2}$

A alimentação adequada nos primeiros meses de vida é fundamental para o crescimento e desenvolvimento saudável da criança. ${ }^{3}$ A Organização Mundial da Saúde (OMS) preconiza o aleitamento materno exclusivo como essencial até os 6 meses de idade, pois é o suficiente para a demanda nutricional. Após esse período, há a necessidade de introduzir novos alimentos, denominados de alimentos complementares, associado à prática do aleitamento materno até os 2 anos de idade, para suprir as necessidades nutricionais da criança. ${ }^{4}$ 
A alimentação complementar é caracterizada pela introdução de qualquer alimento, que não o leite materno, de forma lenta e gradual. ${ }^{3,5}$ No entanto, é importante respeitar o momento adequado para inseri-la, uma vez que a introdução precoce (antes dos 6 meses de vida) é um dos fatores para o desenvolvimento de diarreias, ${ }^{6}$ infecções, desnutrição ${ }^{4}$ e doenças crônicas futuras. ${ }^{7}$

As mães exercem fundamental influência na determinação da alimentação das crianças nessa faixa etária, uma vez que ainda são elas as principais responsáveis pela escolha, compra, preparo e oferta dos alimentos para seus filhos, além de ser, junto à família, a primeira referência para a criança sobre as questões alimentares. ${ }^{8,9}$

É importante identificar as características maternas que possam influenciar de forma negativa na nutrição e saúde dos filhos, para que sejam formuladas estratégias de intervenção para modificar as situações, ${ }^{10}$ pois o público infantil é bastante suscetível ao desenvolvimento de carências nutricionais. ${ }^{3,7}$ Além disso, é importante identificar a situação alimentar atual das crianças para que os dados sirvam de subsídios na elaboração de políticas públicas municipais, a fim de manter ou promover a saúde das crianças.

Nesse sentido, o estudo teve como objetivo avaliar o consumo alimentar de crianças de 6 a 12 meses de idade e sua relação com variáveis sociodemográficas maternas, do município de Arvorezinha, RS.

\section{Metodologia}

Trata-se de um estudo transversal realizado com crianças de 6 a 12 meses de idade e suas respectivas mães da área urbana e rural do município de Arvorezinha, localizado no Estado do Rio Grande do Sul, com 10.595 habitantes. ${ }^{11}$ Para seleção da amostra, foram identificadas as crianças que receberam a vacina Bacillus Calmette - Guérin (BCG) nas Unidades Básicas de Saúde (UBS) do município, no período de setembro de 2014 a maio de 2015, e aquelas que realizam consultas de puericultura nas duas UBS e com registros dos Agentes Comunitários de Saúde do município, resultando em todas as crianças atendidas por um dos fatores citados.

Foram incluídas na pesquisa todas as mães que apresentaram condições de responder às questões e concordaram em participar de forma voluntária perante assinatura do Termo de Consentimento Livre e Esclarecido (TLCE) e, por meio deste, também autorizaram a participação de seu filho. Foram excluídas do estudo todas as crianças que não conviviam com as mães, aquelas cuja mãe não foi encontrada para aplicar o questionário, uma vez que era o sujeito responsável por responder às perguntas, ou por ter excedido o prazo de 12 meses de idade da criança durante o período da coleta. 
A amostra constituída por 75 crianças resultou, em razão dos critérios de exclusão, em 64 crianças, abrangendo 85,33\% do município. A coleta de dados ocorreu no período de setembro a dezembro de 2015 por meio de visita domiciliar e foram coletados pelos Agentes Comunitários de Saúde e por uma acadêmica de nutrição, natural do município em estudo, todos tendo recebido capacitação para aplicar o questionário junto às mães.

Foram avaliadas as variáveis sociodemográficas das crianças: idade em meses, sexo, escolaridade (frequentadores ou não de escolas de Educação Infantil), o tempo de permanência com a mãe (em horas diárias) e o consumo alimentar. As variáveis sociodemográficas das mães foram: idade, a escolaridade, emprego, o tempo do direito à licença-maternidade, a renda mensal (em salários mínimos), zona de procedência (urbana ou rural), beneficiária do programa Bolsa Família (BPBF) e preparo dos alimentos.

O consumo alimentar foi analisado por meio de Formulários de Marcadores de Consumo Alimentar para Indivíduos Menores de Dois anos, disponíveis pelo Sistema de Vigilância Alimentar e Nutricional (SISVAN), ${ }^{2,12}$ sendo que as perguntas remetiam ao dia anterior (ontem). O consumo alimentar foi avaliado pela ingesta de leite materno e não materno, mingau com leite, frutas, iogurte, comida de panela, legumes, vegetais ou fruta de cor laranja ou folhas verdes escuras, verduras de folha, cereais e tubérculos, carnes, fígado, feijão, embutidos, hambúrguer, açúcar ou mel, bebidas adoçadas, biscoito recheado, doces e guloseimas, macarrão instantâneo, salgadinho de pacote ou biscoito salgado.

Para classificar o consumo alimentar como adequado ou não adequado, foram aplicadas as recomendações dos Dez Passos para Alimentação Saudável para Menores de Dois Anos, proposto pelo Ministério da Saúde. ${ }^{13}$ Para analisar o consumo de leite materno e não materno, a introdução de alimentos complementares e o consumo de alimentos industrializados, foram utilizados: o passo 2 (ao completar 6 meses, introduzir de forma lenta e gradual outros alimentos, mantendo o leite materno até os 2 anos de idade ou mais); o passo 3 (ao completar 6 meses, dar alimentos complementares como cereais, tubérculos, carnes, leguminosas, frutas e legumes três vezes ao dia, se a criança estiver em aleitamento materno); e o passo 8 (evitar açúcar, café, enlatados, frituras, refrigerantes, balas, salgadinhos e outras guloseimas, nos primeiros anos de vida. Usar sal com moderação). Foi considerado o consumo alimentar inadequado quando não condizia com os passos supracitados.

Para avaliar o tempo de aleitamento materno, foi questionado se a criança o recebeu no dia anterior. Para as que receberam, foi calculada a mediana de idade através dos dias de vida até o momento da entrevista. Quando as mães relatavam que as crianças estavam desmamadas, questionou-se por quanto tempo houve a oferta de leite materno, em dias, sendo calculado, por fim, a mediana de dias. 
Os dados foram duplamente digitados e analisados no programa Statistical Package for the Social Sciences (SPSS), versão 18.0. Foram construídas tabelas descritivas de frequência simples. Para comparação de médias, utilizou-se o teste t de Student; para comparação de variável categórica, o teste qui-quadrado, considerando o nível de significância a 5\%.

Esta pesquisa foi autorizada pelo Comitê de Ética em Pesquisa da Universidade Federal de Santa Maria (UFSM), seguindo o padrão ético estabelecido pelas Normas e Diretrizes Regulamentadoras da Pesquisa Envolvendo Seres Humanos - Resolução CNS 466/2012, e recebeu como registro o CAAE 48289115.9.0000.5346. A pesquisa também foi autorizada pela Secretaria de Saúde do município de Arvorezinha, RS.

\section{Resultados}

Das 64 crianças avaliadas, 40,6\% ( $\mathrm{n}=26)$ eram do sexo feminino e 59,4\% (n=38), do masculino, a

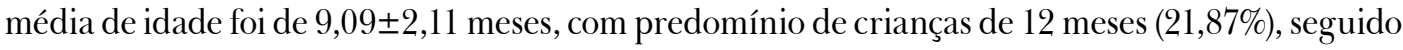
de 8 meses $(20,31 \%)$. Do total da amostra, somente 3,2\% $(\mathrm{n}=2)$ das crianças frequentavam escolas de Educação Infantil. O tempo diário médio de permanência com a mãe foi de 17,91 \pm 8,32 horas.

Em relação às características maternas, a média de idade ficou em 29,45 $\pm 7,11$ anos, a maioria das mães residia na área urbana (74,6\%), a escolaridade predominante foi o Ensino Médio completo $(30,2 \%)$, seguido de Ensino Fundamental incompleto (23,8\%), um quarto eram beneficiárias do programa Bolsa Família $(27,4 \%)$. Metade da amostra relatou trabalhar $(55,2 \%)$, embora, quando questionado o vínculo empregatício, $51,8 \%$ referiram ser dona de casa e não ter renda mensal própria $(45,9 \%)$. Todas as mães que tiveram direito à licença-maternidade $(\mathrm{n}=75,9 \%)$, tiveram por um período de quatro meses, e a maioria relatou ser responsável pelo preparo das refeições em casa $(78,1 \%)$. Quando não era a responsável, a avó da criança preparava $(73,3 \%)$, conforme descrito na Tabela 1. 
Tabela 1. Características sociodemográficas das mães de crianças de 6 meses a 1 ano do município de Arvorezinha, RS (n=64, 2015).

CARACTERÍSTICAS MATERNAS

\begin{tabular}{|c|c|c|}
\hline & $\mathrm{n}$ & $\%$ \\
\hline \multicolumn{3}{|l|}{ Procedência } \\
\hline Urbana & 47 & 74,6 \\
\hline Rural & 16 & 25,4 \\
\hline \multicolumn{3}{|l|}{ Escolaridade } \\
\hline Não estudou & 2 & 3,2 \\
\hline Ensino Fundamental incompleto & 15 & 23,8 \\
\hline Ensino Fundamental completo & 9 & 14,3 \\
\hline Ensino Médio incompleto & 5 & 7,9 \\
\hline Ensino Médio completo & 19 & 30,2 \\
\hline Ensino Superior incompleto & 2 & 3,2 \\
\hline Ensino Superior completo & 11 & 17,5 \\
\hline \multicolumn{3}{|l|}{ Beneficiária do programa Bolsa Família } \\
\hline Sim & 17 & 27,4 \\
\hline Não & 45 & 72,6 \\
\hline \multicolumn{3}{|l|}{ Renda mensal } \\
\hline Nenhuma renda mensal & 28 & 45,9 \\
\hline < 1 Salário Mínimo & 6 & 9,8 \\
\hline 1 a 3 Salários Mínimos & 24 & 39,4 \\
\hline > 3 Salários Mínimos & 3 & 4,9 \\
\hline \multicolumn{3}{|l|}{ Trabalho } \\
\hline Sim & 32 & 55,2 \\
\hline Não & 26 & 44,8 \\
\hline
\end{tabular}




\begin{tabular}{|c|c|c|}
\hline \multicolumn{3}{|c|}{ CARACTERÍSTICAS MATERNAS } \\
\hline & $\mathrm{n}$ & $\%$ \\
\hline \multicolumn{3}{|l|}{ Vinculo empregatício } \\
\hline Carteira de trabalho & 16 & 28,6 \\
\hline Autônoma & 6 & 10,7 \\
\hline Diarista sem carteira de trabalho & 1 & 1,8 \\
\hline Dona de casa & 29 & 51,8 \\
\hline Funcionária Pública & 3 & 5,4 \\
\hline Aposentada & 1 & 1,8 \\
\hline \multicolumn{3}{|l|}{ Direito à licença-maternidade } \\
\hline Sim & 41 & 75,9 \\
\hline Não & 13 & 24,1 \\
\hline \multicolumn{3}{|l|}{ Preparo das refeições } \\
\hline Mãe da criança & 50 & 78,1 \\
\hline Outro & 14 & 21,9 \\
\hline \multicolumn{3}{|l|}{ Quem prepara a refeição (Outro) } \\
\hline Avó & 11 & 73,3 \\
\hline Babá/cuidador & 1 & 6,7 \\
\hline Marido & 2 & 13,3 \\
\hline Esposa e marido & 1 & 6,7 \\
\hline
\end{tabular}

A Tabela 2 demonstra o consumo alimentar das crianças, no dia anterior à coleta de dados, relacionado com a média de idade das mesmas. 
Tabela 2. Porcentual do consumo alimentar no dia anterior à coleta de dados, conforme a média de idade das crianças em meses no município de Arvorezinha, $\operatorname{RS}(\mathrm{n}=64,2015)$.

\begin{tabular}{|c|c|c|c|}
\hline Consumo alimentar & $\begin{array}{c}\text { Porcentual } \\
\text { (\%) de } \\
\text { consumo }\end{array}$ & $\begin{array}{c}\text { Média de idade } \\
\text { da criança em } \\
\text { meses }\end{array}$ & $\mathrm{p}$ \\
\hline \multicolumn{4}{|l|}{ Mingau com leite } \\
\hline Sim & 32,81 & $9,62 \pm 2,13$ & \multirow{2}{*}{0,165} \\
\hline Não & 67,19 & $8,84 \pm 2,06$ & \\
\hline \multicolumn{4}{|c|}{ Fruta inteira/amassada/pedaço } \\
\hline Sim & 87,30 & $9,09 \pm 2,08$ & \multirow{2}{*}{0,845} \\
\hline Não & 12,70 & $9,25 \pm 2,49$ & \\
\hline \multicolumn{4}{|l|}{ Iogurte } \\
\hline Sim & 54,1 & $9,58 \pm 2,10$ & \multirow{2}{*}{0,036} \\
\hline Não & 45,90 & $8,46 \pm 1,89$ & \\
\hline \multicolumn{4}{|l|}{ Comida de sal } \\
\hline Sim & 95,31 & $9,23 \pm 2,06$ & \multirow{2}{*}{0,001} \\
\hline Não & 4,69 & $6,33 \pm 0,57$ & \\
\hline \multicolumn{4}{|l|}{ Legumes } \\
\hline Sim & 70,31 & $9,13 \pm 1,94$ & \multirow[b]{2}{*}{0,837} \\
\hline Não & 29,69 & $9,0 \pm 2,49$ & \\
\hline \multicolumn{4}{|c|}{ Vegetal ou fruta alaranjada ou folhas verdes escuras } \\
\hline Sim & 57,81 & $9,19 \pm 1,94$ & \multirow{2}{*}{0,675} \\
\hline Não & 42,19 & $8,96 \pm 2,34$ & \\
\hline \multicolumn{4}{|l|}{ Verdura de folha } \\
\hline Sim & 15,64 & $10,10 \pm 2,02$ & \multirow{2}{*}{0,100} \\
\hline Não & 84,36 & $8,91 \pm 2,08$ & \\
\hline \multicolumn{4}{|l|}{ Carne } \\
\hline Sim & 71,88 & $9,70 \pm 1,96$ & \multirow{2}{*}{0,000} \\
\hline Não & 28,12 & $7,56 \pm 1,65$ & \\
\hline \multicolumn{4}{|l|}{ Fígado } \\
\hline Sim & 4,76 & $10,67 \pm 2,30$ & \multirow{2}{*}{0,195} \\
\hline Não & 95,24 & $9,03 \pm 2,09$ & \\
\hline
\end{tabular}




\begin{tabular}{|c|c|c|c|}
\hline \multicolumn{4}{|l|}{ Feijão } \\
\hline Sim & 57,38 & $9,11 \pm 2,08$ & \multirow{2}{*}{0,946} \\
\hline Não & 42,62 & $9,08 \pm 2,17$ & \\
\hline \multicolumn{4}{|c|}{ Arroz/batata/inhame/mandioca/farinha/macarrão } \\
\hline Sim & 90,48 & $9,30 \pm 2,02$ & \multirow{2}{*}{0,029} \\
\hline Não & 9,52 & $7,33 \pm 2,33$ & \\
\hline \multicolumn{4}{|c|}{ Hambúrguer ou embutidos } \\
\hline $\operatorname{Sim}$ & 7,94 & $9,40 \pm 2,40$ & \multirow{2}{*}{0,765} \\
\hline Não & 92,06 & $9,10 \pm 2,10$ & \\
\hline \multicolumn{4}{|c|}{ Bebidas adoçadas } \\
\hline $\operatorname{Sim}$ & 38,1 & $9,50 \pm 2,12$ & \multirow{2}{*}{0,200} \\
\hline Não & 61,9 & $8,79 \pm 2,08$ & \\
\hline \multicolumn{4}{|c|}{ Biscoito recheado/doces/guloseimas } \\
\hline Sim & 29,69 & $9,21 \pm 2,17$ & \multirow{2}{*}{0,776} \\
\hline Não & 70,31 & $9,04 \pm 2,09$ & \\
\hline \multirow{2}{*}{\multicolumn{4}{|c|}{$\begin{array}{l}\text { Macarrão instantâneo/salgadinho de pacote/biscoito } \\
\text { salgado }\end{array}$}} \\
\hline & & & \\
\hline $\operatorname{Sim}$ & 17,46 & $10 \pm 1,84$ & \multirow{2}{*}{0,127} \\
\hline Não & 82,54 & $8,92 \pm 2,14$ & \\
\hline
\end{tabular}

P* teste t de student comparando a média de idade das crianças com o consumo ou não do alimento.

Quando relacionado o consumo de alimentos com a idade da criança, observa-se na Tabela 2 que o consumo no dia anterior de iogurte $(\mathrm{p}=0,036)$, comida de sal $(\mathrm{p}=0,001)$, carne $(\mathrm{p}=0,000)$ e arroz ou batata ou inhame ou mandioca ou farinha ou macarrão $(p=0,029)$ se deu entre as crianças com maior idade em meses, quando comparado com as crianças que não comeram estes alimentos no dia anterior.

Sobre o consumo de leite materno, foi possível identificar que 37 crianças $(57,81 \%)$ estavam em aleitamento materno, pois o receberam no dia anterior, com média de idade de 8,92 $\pm 2,10$ meses. As crianças que não consumiram o leite materno no dia anterior já estavam desmamadas à época da pesquisa, sendo que a mediana de dias de aleitamento materno das mesmas foi de 135 dias. Foi constatado que $17,19 \%$ das crianças receberam mel ou melado ou açúcar ou rapadura antes dos 6 meses de idade.

A Tabela 3 compara o porcentual do consumo alimentar das crianças no dia anterior à coleta de dados com as variáveis maternas de interesse no estudo. 


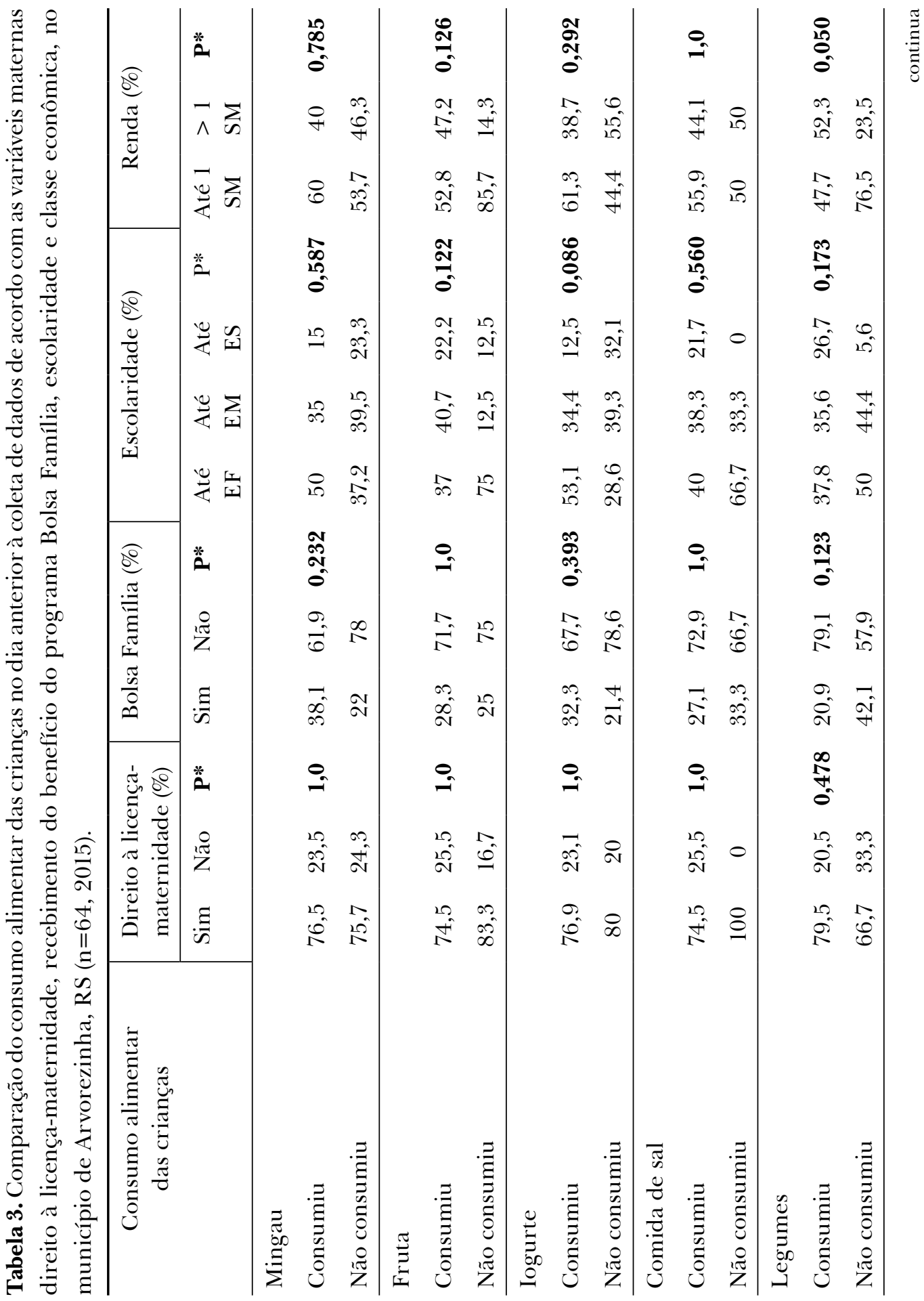




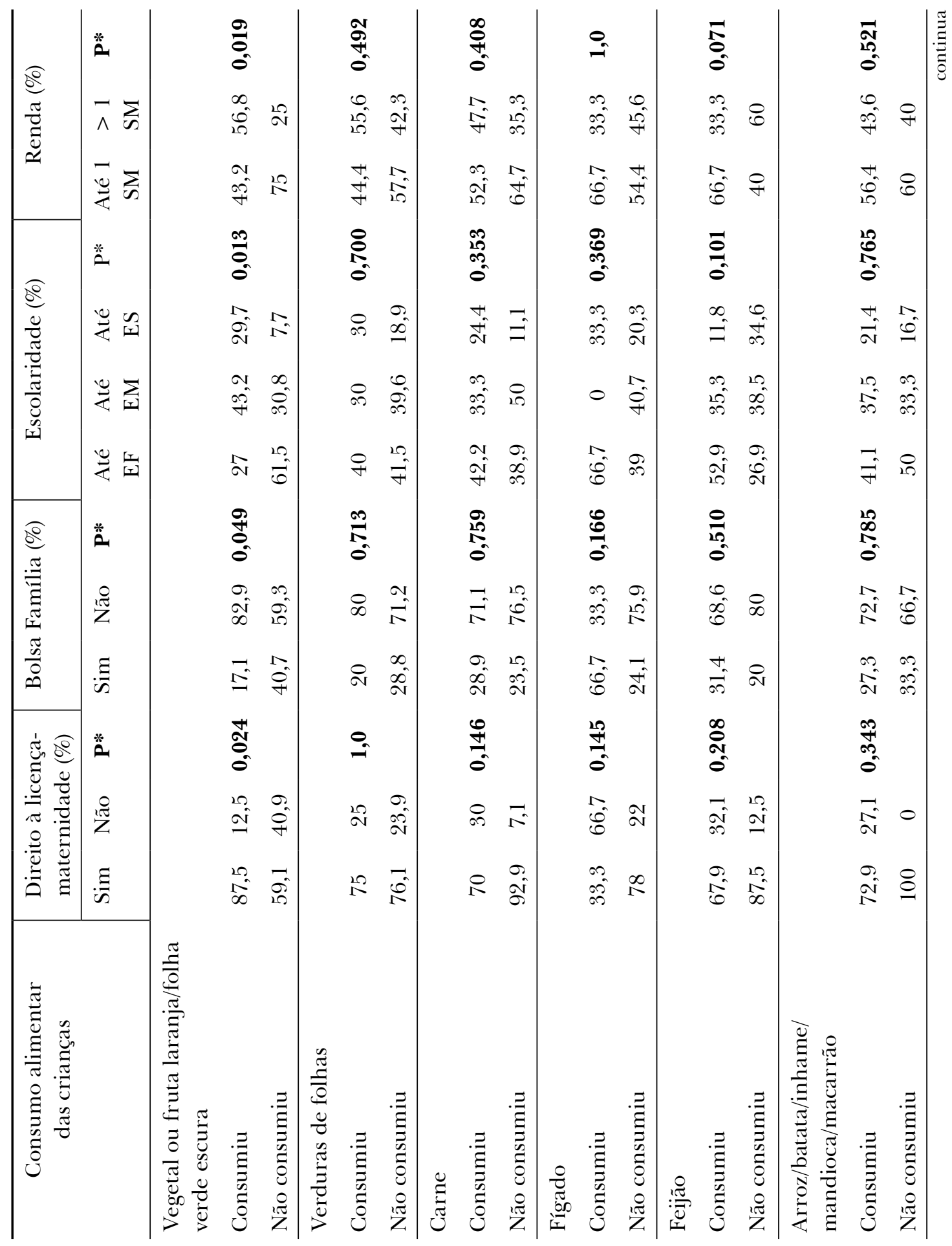




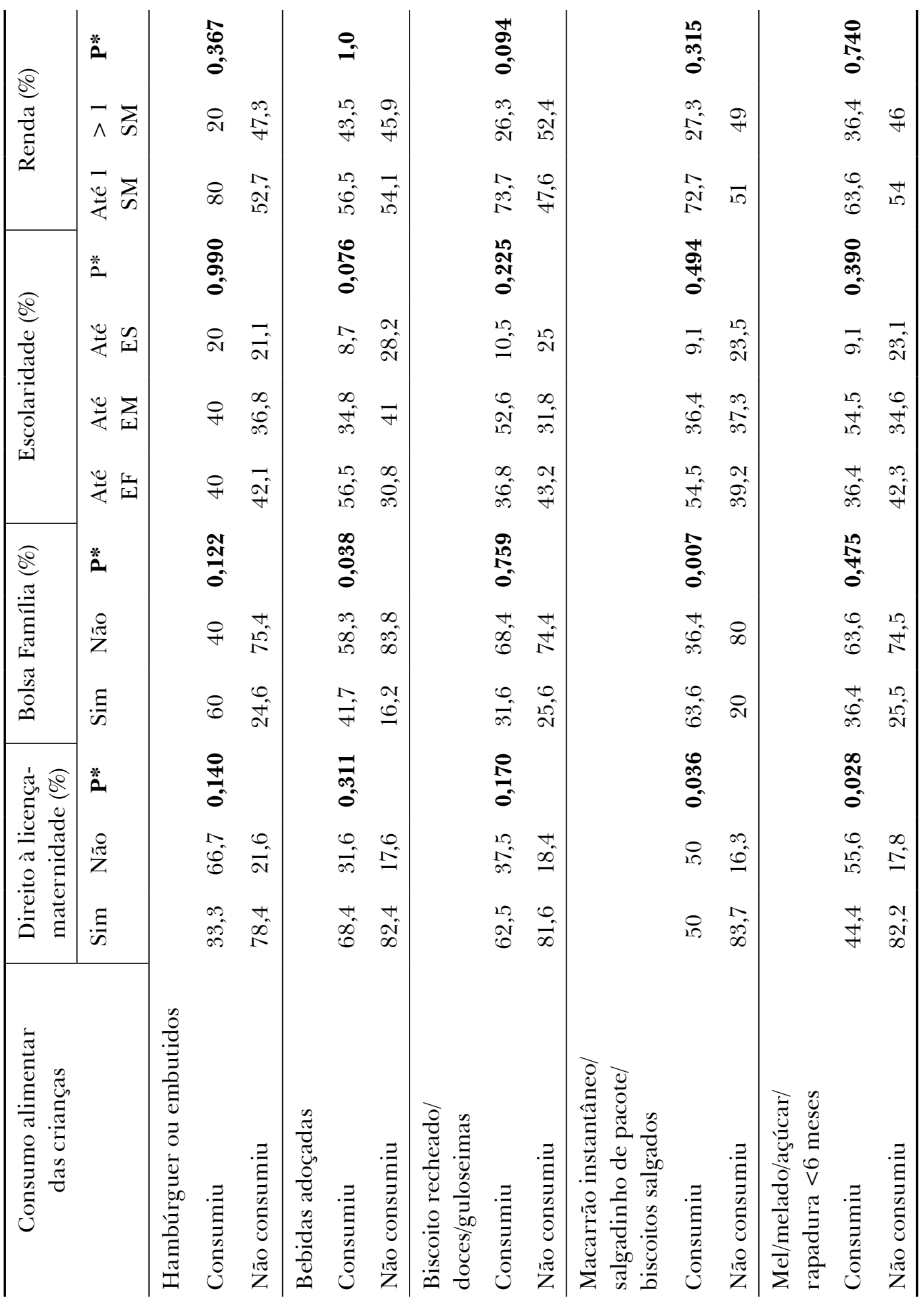


Ao comparar o consumo alimentar das crianças com as características maternas, verificou-se que os filhos das mães que tiveram direito à licença-maternidade consumiram fruta $(p=0,024)$, não consumiram macarrão instantâneo, salgadinho de pacote ou biscoito salgado $(p=0,036)$ e mel, melado, açúcar ou rapadura antes dos 6 meses de idade $(\mathrm{p}=0,028)$.

Em relação ao recebimento do benefício do programa Bolsa Família, os filhos das mães que não eram beneficiárias do programa consumiram mais vegetais ou fruta cor alaranjada ou folhas verdes escuras que as demais $(p=0,049)$. Entretanto, observou-se maior consumo de macarrão instantâneo, salgadinho de pacote ou biscoitos salgados e bebidas adoçadas $(p=0,038)$ entre os filhos de mães que eram beneficiárias do programa.

Ainda, os filhos cujas mães estudaram até o Ensino Médio consumiram mais vegetal ou fruta cor alaranjada ou folhas verdes escuras $(p=0,013)$, e os filhos cujas mães recebiam mais que um salário mínimo mensal consumiram mais legumes $(\mathrm{p}=0,050)$ e vegetal ou fruta cor alaranjada ou folhas verdes escuras $(p=0,019)$.

Por fim, o estudo também demonstrou que os filhos das mães com média de idade de 27 anos consumiram mais iogurte ( $\mathrm{p}=0,006)$; com 25 anos, mais verdura de folhas $(\mathrm{p}=0,034)$; e com 28 anos, mais feijão $(p=0,019)$, não exposto em tabelas.

\section{Discussão}

Conhecer o padrão alimentar da população é fundamental para criar ações de atenção à saúde. Os protocolos que avaliam o consumo alimentar infantil disponíveis pelo SISVAN questionam o consumo alimentar no dia anterior, o que possibilita uma diminuição em erros por viés de memória. São instrumentos que simplificam a coleta e análise dos dados, além de ser fácil e rápida a aplicação, para que qualquer profissional de saúde o utilize e avalie rotineiramente o consumo alimentar infantil. ${ }^{12}$

O presente estudo demonstrou que a média de idade das crianças que consumiram os alimentos iogurte, carne, comida de sal, arroz ou batata ou inhame ou mandioca, farinha ou macarrão (sem ser instantâneo) foi maior em comparação à média de idade das crianças que não consumiram estes alimentos, evidenciando maior consumo em torno dos 9 meses. Além disso, foi observada a alta frequência na ingesta de frutas, comida de sal, legumes, arroz ou batata ou inhame ou mandioca, e farinha ou macarrão (sem ser instantâneo), e baixa frequência na ingesta de mingau com leite, verdura de folha, fígado, hambúrguer ou embutidos, biscoito doce, recheados e guloseimas, macarrão instantâneo, salgadinho de pacote e biscoito salgado na alimentação das crianças participantes.

O consumo alimentar das crianças relacionado ao perfil sociodemográfico materno apresentou relação estatística significativa entre as variáveis idade materna, direito à licença-maternidade, 
ser beneficiária do programa Bolsa Família, ter estudado até o Ensino Médio e ter renda mensal própria acima de um salário mínimo.

Os resultados obtidos demonstram a situação alimentar de crianças de 6 meses a 1 ano de idade do município de Arvorezinha, RS, tornando este estudo inédito no município e no Estado do Rio Grande do Sul, sendo o primeiro a utilizar a atualização do protocolo do SISVAN ${ }^{12}$ para crianças menores de 1 ano, até o presente momento.

O consumo alimentar infantil é bastante discutido pela literatura atual através de dados obtidos por Recordatório Alimentar de 24 horas e Questionários de Frequência Alimentar. Somente o estudo de Coelho et. al., ${ }^{10}$ realizado em Diadema, São Paulo, com 350 crianças menores de 24 meses, utilizou o protocolo do SISVAN² para realizar a coleta de dados, sendo o único publicado. Eles verificaram que alimentos como o açúcar, mel ou melado, papa salgada e leite não humano são oferecidos antes dos 6 meses de idade, abandonando o aleitamento materno exclusivo, além do consumo insuficiente de frutas, legumes e vegetais, carne e feijão entre 6 e 23 meses, pois não foram ofertados na frequência recomendada pelo Guia Alimentar para Crianças Menores de Dois Anos. ${ }^{13}$

O nosso estudo apresenta semelhanças em relação ao consumo de açúcar, mel ou melado antes dos 6 meses de idade e no consumo pouco frequente de frutas, legumes, vegetais, carne e feijão, contrariando o terceiro passo do guia. ${ }^{13}$

Os alimentos com maior frequência de ingesta pelas crianças menores de 1 ano foram as frutas, legumes, cereais, comida de sal e carnes. Hipóteses para isso são as questões culturais da população, que prezam pelo consumo de alimentos in natura na primeira infância, pelo acesso facilitado ao alimento in natura, podendo ser adquirido direto com o produtor rural, bem como as recomendações dos profissionais da saúde na atenção básica, que estimulam o aleitamento materno e a alimentação complementar conforme as recomendações do Ministério da Saúde. ${ }^{13}$

O oitavo passo do Guia Alimentar para Menores de Dois Anos ${ }^{13}$ recomenda evitar açúcar, café, enlatados, frituras, refrigerantes, balas, salgadinhos e outras guloseimas, nos primeiros anos de vida, bem como usar sal com moderação. O nosso estudo identificou o consumo destes alimentos no processo da alimentação complementar antes de 1 ano de idade, sendo as bebidas adoçadas, iogurte, balas, guloseimas ou biscoitos doces os mais ofertados, apesar de não ser com alta frequência.

Em relação ao aleitamento materno, a média de idade das crianças que o receberam no dia anterior foi de 270 dias, o que pode estar relacionado com a maior prevalência de crianças com

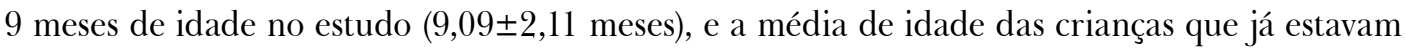
desmamadas foi de 128,62 \pm 96,36 dias. Ambas ficaram abaixo da média brasileira, correspondente a 341,6 dias, ${ }^{14}$ e não atenderam às recomendações do segundo passo do Guia Alimentar para Menores de Dois Anos. ${ }^{13}$ Condizendo com nosso estudo, Saldiva et al. ${ }^{15}$ verificaram em São Paulo que apenas $50 \%$ das crianças de 6 a 12 meses receberam o leite materno no dia anterior à coleta de dados. 
As variáveis maternas também demonstraram associações no consumo alimentar infantil. Molina et al. ${ }^{16}$ verificaram que a baixa escolaridade materna resulta na alimentação da criança de baixa qualidade e Campagnolo et al. ${ }^{17}$ identificaram que o maior nível de escolaridade materna tem influenciado positivamente no consumo de legumes ou verduras e carnes entre os lactentes. Em nosso estudo, constatou-se associação no consumo de vegetal ou fruta laranja ou folhas verdes escuras por crianças cujas mães estudaram até o Ensino Médio, o que se justifica pelo nível de instrução e orientação maior.

Outro fator importante a ser analisado quando se busca identificar influências no consumo alimentar infantil é a renda. O nosso estudo corrobora os resultados do estudo de Drewnowski $\&$ Specter, ${ }^{18}$ mas a renda da família mostrou associação com o consumo de frutas, vegetais ou verduras, pois as de baixa renda consumiram menores quantidades destes alimentos. O elevado custo das frutas e vegetais ou verduras e o difícil acesso a estes alimentos podem ser fatores que impossibilitem a família a tê-los diariamente nas refeições.

Côrrea et al. ${ }^{19}$ verificaram que a renda mensal materna menor de um salário mínimo tem associação com o maior consumo de carne, doces e panificação pelas crianças menores de dois anos. O estudo de Silva et al. ${ }^{20}$ demonstrou que, conforme a renda da família aumentava, proporcionava o aumento também no consumo de alimentos industrializados, ricos em gordura e açúcares. O nosso estudo não apresentou diferença significativa da renda com o consumo de alimentos industrializados. Contudo, o consumo de bebidas adoçadas evidenciou associação com mães não beneficiárias do programa Bolsa Família, estando relacionado, indiretamente, à renda.

No presente estudo foi verificada baixa frequência da ingesta de frutas, verduras e vegetais verdes escuros por crianças beneficiárias do programa Bolsa Família e maior consumo de alimentos industrializados, como macarrão instantâneo, salgadinho de pacote, biscoitos salgados e hambúrgueres ou embutidos, não sendo considerado um consumo adequado para a faixa etária. ${ }^{13}$ Tais resultados corroboram com os encontrados por Saldiva et al. ${ }^{21}$ Entretanto, são alimentos mais baratos e as beneficiárias do programa Bolsa Família são pessoas de menor poder aquisitivo, o que influencia na compra de alimentos de acordo com a renda. O porcentual de beneficiárias achava-se abaixo de $50 \%$ e foram encontrados poucos estudos ${ }^{10,20}$ publicados referentes ao consumo alimentar infantil e participação no programa.

Nosso estudo também indicou associação da idade materna e do direito à licença-maternidade com o consumo de alimentos pelas crianças. Os filhos, cujas mães apresentaram idade menor que 28 anos, demonstraram consumir com maior frequência iogurte, verdura de folha e feijão. Coelho et. al. ${ }^{10}$ verificaram maior consumo de leite materno quando as mães eram mais velhas e Saldiva et al. ${ }^{15}$ maior consumo de comida de panela quando as mães tinham menos de 20 anos. Não foram encontradas publicações que demonstrassem associações semelhantes a deste artigo, apesar de vários autores s,9,19,22 $^{7}$ pesquisarem a idade materna e o consumo alimentar infantil. 
Em relação ao direito à licença-maternidade, todas as mães que o tiveram foi por um período de quatro meses, e verificou-se maior consumo de vegetal, fruta laranja ou folhas verdes escuras, menor consumo de macarrão instantâneo, biscoito salgado ou salgadinho de pacote e menor consumo de mel, melado, açúcar ou rapadura antes dos 6 meses de idade. Esses dados nos mostram que a permanência da mãe em casa, com o filho, proporcionou uma alimentação mais saudável e adequada para o mesmo. Além disso, no questionário, o consumo de vegetal, fruta laranja ou folhas verdes escuras conformava uma única questão, o que nos leva a concluir, pelo tempo de licença-maternidade e, consequentemente, pela idade da criança, que o consumo, na maior parte, referia-se às frutas.

Viera et al. ${ }^{22}$ afirmam que a introdução de alimentos complementares ocorre, geralmente, após o término da licença-maternidade pelas mães. Atualmente, os estudos ${ }^{16,23,24}$ demonstram associações entre a prática do aleitamento materno e o direito à licença-maternidade, não havendo associações com a alimentação complementar. Mas é importante estudá-la, visto que se observa constantemente a alimentação complementar prematura.

Contudo, segundo os dados do estudo de Dame et al., ${ }^{25}$ no Rio Grande do Sul há uma baixa cobertura (61,3\% dos municípios) no preenchimento dos dados do SISVAN, devido à não utilização do sistema e de materiais disponíveis, como os protocolos ${ }^{2,12}$ empregados nesta pesquisa, pelos poderes públicos municipais. Outros estudos que utilizaram o SISVAN ${ }^{26-28}$ verificaram apenas o estado nutricional, através da análise antropométrica ou relacionado ao aleitamento materno. No caso do município de Arvorezinha, RS, o SISVAN é usado apenas para avaliar peso e altura dos beneficiários do programa Bolsa Família.

Como limitações do estudo, uma dificuldade encontrada foi a mudança de domicílio, atrapalhando a localização dos admissíveis entrevistados, e o possível viés de memória das mães. Também se percebeu, através da fala contraditória, que algumas mães omitiram informações quanto à introdução e frequência de alguns alimentos. No entanto, o estudo foi importante para identificar alimentos que estavam e não estavam sendo introduzidos no processo da alimentação complementar, o que deve estimular os gestores a buscarem medidas efetivas para modificar a situação encontrada. Ainda, a fim de que haja adesão ao uso dos protocolos, é necessária a capacitação e sensibilização dos profissionais de saúde.

Os resultados nos levam a concluir que o consumo alimentar infantil nesta faixa etária é baseado em alimentos in natura, mas as mães não seguem as recomendações do Ministério da Saúde ${ }^{3}$ quanto à introdução de novos alimentos e permanência do aleitamento materno até os 2 anos de idade, devendo fortemente ser incentivado o aleitamento materno e a alimentação complementar, conforme as recomendações, desde a gestação. Além disso, as variáveis maternas idade, direito à licença-maternidade, Bolsa Família, escolaridade e renda interferem no consumo alimentar dos filhos. 


\section{Colaboradores}

Coradi FB, Bottaro SM e Kirsten VR trabalharam em todas as etapas, desde a concepção do estudo até a revisão da versão final do artigo.

Conflito de interesses: Os autores declaram não haver conflito de interesses.

\section{Referências}

1. Brasil. Vigilância Alimentar e Nutricional - SISVAN: orientações básicas para a coleta, processamento, análise de dados e informação em serviços de saúde. Brasília: Ministério da Saúde; 2004.

2. Brasil. Ministério da Saúde. Protocolos do Sistema de Vigilância Alimentar e Nutricional: SISVAN na assistência à saúde. Brasília: Ministério da Saúde; 2008.

3. Brasil. Ministério da Saúde. Organização Pan Americana da Saúde. Guia alimentar para crianças menores de dois anos. Brasília: Ministério da Saúde; 2002.

4. World Organization Health. The optimal duration of exclusive breastfeeding: a systematic review. Geneva: WHO; 2001.

5. Brasil. Ministério da Saúde. Saúde da criança: nutrição infantil: aleitamento materno e alimentação complementar. Brasília: Ministério da Saúde; 2009.

6. World Organization Health. UNICEF. Complementary feeding of young children in developing countries: a review of current scientific knowledge. Geneva: World Health Organization; 1998.

7. Caetano MC, Ortiz TTO, Silva SGL, Souza FIS, Sarni ROS. Complementary feeding: inappropriate practices in infants. J Pediatr. 2010; 86:86-93.

8. Cobelo AW. O papel da família no comportamento alimentar e nos transtornos alimentares. In: Philippi ST, Alvarenga M. Transtornos alimentares: uma visão nutricional. São Paulo: Manole; 2004. p. 119-129.

9. Francis LA, Birch LL. Maternal influences on daughters restrained eating behavior. Health Psychol 2005; 24:548-554.

10. Coelho LC, Asakura L, Sacks A, Erbert I, Novaes CRL, Gimeno SGA. Sistema de Vigilância Alimentar e Nutricional/SISVAN: conhecendo as práticas alimentares de crianças menores de 24 meses. Ciênc Saúde Coletiva 2015; 20:727-738.

11. Instituto Brasileiro de Geografia e Estatística. Rio Grande do Sul: Arvorezinha. Informações completas. População estimada em 2015. IBGE, 2015. [acesso em: 13 jul. 2016]. Disponível em: http://cidades.ibge.gov.br/xtras/perfil.php?lang=\&codmun=430140\&search $=||$ infogr\%E1ficos:informa $\% \mathrm{E} 7 \%$ F5es-completas

12. Brasil. Ministério da Saúde. Secretaria de Atenção à Saúde. Orientações para avaliação de marcadores de consumo alimentar na atenção básica [recurso eletrônico] Brasília: Ministério da Saúde; 2015.

13. Brasil. Ministério da Saúde. Secretaria de Atenção à Saúde. Dez passos para uma alimentação saudável: guia alimentar para crianças menores de dois anos: um guia para o profissional da saúde na atenção básica. 2 ed. Brasília: Ministério da Saúde; 2010. 
14. Brasil. Ministério da Saúde. Secretaria de Atenção a Saúde. II Pesquisa de Prevalência de Aleitamento Materno nas Capitais Brasileiras e Distrito Federal. Brasília: Editora do Ministério da Saúde; 2009.

15. Saldiva SRDM, Escuder MM, Mondini L, Levy RB, Venancio SI. Feeding habits of children aged 6 to 12 months and associated maternal factors. J Pediatr. 2007; 83:53-58.

16. Molina MCB, López PM, Faria CP, Cade NV, Zandonade E. Preditores socioeconômicos da qualidade da alimentação de crianças. Rev Saúde Públ. 2010; 44:785-732.

17. Campagnolo PDB, Louzada MLC, Silveira EL, Vitolo MR. Práticas alimentares no primeiro ano de vida e fatores associados em amostra representativa da cidade de Porto Alegre, Rio Grande do Sul. Rev Nutr. 2012; 25:431-439.

18. Drewnowski A, Specter E. Poverty and obesity: the role of energy density and energy costs. Am J Clin Nutr. 2004; 79:6-16.

19. Corrêa EM, Corso ACT, Moreira EAM, Kazapi IAM. Alimentação complementar e características maternas de crianças menores de dois anos de idade em Florianópolis (SC). Rev Paul Pediatr. 2009; 27:258-264.

20. Silva RCR, Assis AMO, Szarfarc SC, Pinto EJ, Costa LCC, Rodrigues LC. Iniquidades socioeconômicas na conformação dos padrões alimentares de crianças e adolescentes. Rev Nutr. 2012; 25:451-461.

21. Saldiva SRDM, Silva LFF, Saldiva PHN. Avaliação antropométrica e consumo alimentar em crianças menores de cinco anos residentes em um município da região do semiárido nordestino com cobertura parcial do programa bolsa família. Rev Nutr. 2010; 23:221-229.

22. Vieira GO, Silva LR, Vieira TO, Almeida JAG, Cabral VA. Hábitos alimentares de crianças menores de 1 ano amamentadas e não-amamentadas. J Pediatr. 2004; 80:411-416.

23. Ferreira GR, D’Artibale EF, Bercini LO. Influência da prorrogação da licença maternidade para seis meses na duração do aleitamento materno exclusivo. Rev Min Enf. 2013; 17:162-168.

24. Brasileiro AA, Ambrosano GMB, Marba STM, Possobon RF. A amamentação entre filhos de mulheres trabalhadoras. Rev Saúde Públ. 2012; 46:642-648.

25. Dame PKV, Pedroso MRO, Marinho CL, Gonçalves VM, Duncan BB, Fisher PD, et al. Sistema de Vigilância Alimentar e Nutricional (SISVAN) em crianças do Rio Grande do Sul, Brasil: cobertura, estado nutricional e confiabilidade dos dados. Cad Saúde Pública 2011; 27:2155-2165.

26. Felisbino-Mendes MS, Campos MD, Lana FCF. Avaliação do estado nutricional de crianças menores de 10 anos no município de Ferros, Minas Gerais. Rev Esc Enferm USP 2010; 44:257-265.

27. Pereira AS, Peixoto NGA, Nogueira Neto JF, Lanzilotti HS, Soares EA. Estado nutricional de préescolares de creche pública: um estudo longitudinal. Cad Saúde Coletiva 2013; 21:140-147.

28. Silva DAS, Nunes HEG. Prevalence of underweight, overweight and obesity in poor children from Mato Grosso do Sul. Rev Bras Epidemiol. 2015; 18:466-475.

Recebido: $27 / 03 / 2017$

Revisado: 13/07/2017

Aceito: 25/08/2017 